Research Article

\title{
Diffuse Scattering Directive Model Parameterization Method for Construction Materials at mmWave Frequencies
}

\author{
Ming-Hao Ren, ${ }^{1}$ Xi Liao $\mathbb{D}^{1,2}$ Jihua Zhou, ${ }^{3}$ Yang Wang, ${ }^{1}$ Yu Shao, ${ }^{1}$ Shasha Liao, ${ }^{1,2}$ \\ and Jie Zhang ${ }^{4}$ \\ ${ }^{1}$ Chongqing Key Laboratory of Mobile Communications Technology, \\ Engineering Research Center of Mobile Communications of the Ministry of Education, \\ School of Communication and Information Engineering, Chongqing University of Posts and Telecommunications, \\ Chongqing 400065, China \\ ${ }^{2}$ State Key Laboratory of Millimeter Waves, Southeast University, Nanjing 210096, Jiangsu, China \\ ${ }^{3}$ Chongqing Jinmei Communication Co., Ltd., Chongqing 400030, China \\ ${ }^{4}$ Department of Electronic and Electrical Engineering, University of Sheffield, Sheffield, S10 2TN, UK
}

Correspondence should be addressed to Xi Liao; liaoxi@cqupt.edu.cn

Received 20 June 2020; Revised 2 September 2020; Accepted 12 December 2020; Published 23 December 2020

Academic Editor: Francesco D'Agostino

Copyright $\odot 2020$ Ming-Hao Ren et al. This is an open access article distributed under the Creative Commons Attribution License, which permits unrestricted use, distribution, and reproduction in any medium, provided the original work is properly cited.

The determination for diffuse scattering model parameters is of critical importance to improve the accuracy of prediction and analysis for millimeter-wave radio propagation. In this paper, a reliable parameterization method with a simplified tuning procedure for a diffuse scattering model is proposed and validated based on measurements and ray-tracing simulations. Typical construction materials are measured from $40 \mathrm{GHz}$ to $50 \mathrm{GHz}$, and the complex permittivity is estimated by the propagation coefficients match method. The directive model, which can better characterize the scattering patterns of construction materials in this paper, is adopted to calibrate the ray-tracing simulation. Model parameterization is performed and simulated results with the optimal model parameters show distinct accuracy improvement.

\section{Introduction}

As a typical scenario of millimeter-wave (mmWave) network deployments, indoor communication encounters great challenges in complex propagation environments with various materials [1]. Radio propagation in indoor environments can be studied using stochastic and deterministic based approaches. As an accurate deterministic simulation method with detailed and reliable environment description, ray tracing (RT) has been widely adopted to catch the multipath information for predicting and analyzing channel propagation characteristics in various scenarios [2]. Since the surface irregularities of some materials are comparable to the mmWave wavelengths, the impacts of diffuse scattering (DS) in mmWave bands become more severe than conventional wireless communication bands. A few studies have demonstrated that DS is a significant propagation mechanism for characterizing the multipath components of radio signals in mmWave bands and beyond [3, 4]. Although RT simulation has been validated to be an effective method for predicting the specular reflection, transmission, diffraction, and multiple propagation, it cannot predict the dense multipath components from DS propagation, which leads to the inability to accurately analyze mmWave channel characteristics. Therefore, the DS propagation phenomena must be studied and modeled to calibrate the RT simulation for accurate channel analysis in mmWave bands.

In the past years, many measurement campaigns and researches have been launched for the sake of acquiring an indepth knowledge of scattering characteristics at several very promising mmWave frequencies in order to predict mmWave propagation and analyze channel characteristics in indoor and outdoor environments, which is essential for the deployment of future mmWave communication systems. For 
example, the interaction of radio waves with a typical office building and resulting scattering characteristics is analyzed at $60 \mathrm{GHz}$ and $70 \mathrm{GHz}$ in [5] through measurements and RT simulations on a scale model of the building. Results show that scattering from buildings can be modeled neglecting the internal structure, while proper modeling of nonspecular propagation is necessary. In [6], DS has been demonstrated to make a noticeable contribution to the received signal power of non-line-of-sight links in mmWave bands through the ray launching tool with the DS model, and the contribution can be higher with increasing frequency. Despite DS measurement campaigns conducted at $28 \mathrm{GHz}, 70 \mathrm{GHz}$, and more recently at $60 \mathrm{GHz}$, the propagation characteristics of DS in $\mathrm{mmW}$ ave bands still need further investigations, especially in the band from $40 \mathrm{GHz}$ to $50 \mathrm{GHz}$.

With respect to researches on DS propagation modeling, three DS models characterizing different scattering patterns have been proposed and validated in [7] based on the measurement and RT simulation, including the Lambertian model, the directive model, and the backscattering lobe model. The influence of typical objects in rural railway environment to the mmWave propagation channel is analyzed in [8], and the directive scattering model is adopted in RT simulation. In [9], the three-dimensional characteristics of DS are analyzed by performing electromagnetic simulation and measurements on building materials, and results show that DS is more evident in the backward half-space than in the forward. Scattering mechanisms in Terahertz bands have been investigated in [10] through the directive scattering model and radar cross-section model. The measured results for drywall show that received power in the reflected direction is greater at larger incident angles, while the backscattering power becomes weaker.

In order to determine the DS characteristics in complex environments for accurate channel analysis, the appropriate set of DS model parameters should be estimated for different construction materials to calibrate the RT simulation. In [11], the tuning procedure for DS model parameterization is proposed based on measurements at $60 \mathrm{GHz}$ and the optimal parameters are included in the RT tool for channel analysis with dense components. The procedure, however, is relatively complex because several groups of comparisons are required to determine the appropriate range of scattering coefficient. Moreover, the dielectric parameter estimation for materials under test (MUT) is not involved, which can impact the accuracy of model parameterization. In addition, the lack of various types of materials and model parameterization in other mmWave bands restricts the application of DS model in RT simulation. Therefore, a multicoefficient estimation method for dielectric parameters of rough materials and effect level based parameterization method for DS models are proposed in [12]. The rough rock commonly used as a building material and the plastic carpet considered as indoor decorative material are parameterized, respectively, for the directive model and double-lobe model. Furthermore, a complete methodology for DS model parameterization and RT calibration is proposed and validated in this paper, and more accurate parameter sets are obtained in terms of dielectric parameters, propagation coefficients, and
DS model parameters for two additional construction materials, i.e., granite and marble, at the mmWave band spanning from $40 \mathrm{GHz}$ to $50 \mathrm{GHz}$.

The remaining sections of this paper are organized as follows. In Section 2, measurement campaigns are described and the estimation for dielectric parameters is explained. Then, the DS model parameterization method is proposed and numerical results are analyzed in Section 3. The parameterization method is validated for $\mathrm{RT}$ calibration in Section 4. Finally, conclusions are addressed in Section 5.

\section{Measurement and Dielectric Parameters}

2.1. Measurement Description. The measurement for typical construction materials is performed from $40 \mathrm{GHz}$ to $50 \mathrm{GHz}$ containing 1001 sweep frequency points in an indoor scenario as the essential link for DS model parameterization, which can also provide the primary understanding of DS propagation characteristics. The measurement system, as shown in Figure 1, consists of an N5235B vector network analyzer operated from $100 \mathrm{MHz}$ to $50 \mathrm{GHz}$ (the dynamic range is $122 \mathrm{~dB}$ ), which is used to collect the S21 data in the frequency domain, a pair of wideband horn antennas (working at 40-60 GHz), respectively, as the transmitter (Tx) and receiver $(\mathrm{Rx})$, a pair of connecting cables which has a length of $3 \mathrm{~m}$ (the total loss is $20 \mathrm{~dB}$ at $45 \mathrm{GHz}$ ), and MUT, i.e., granite and marble, which are commonly used to cover the surface of floor and wall.

The reflection coefficient is mainly related to frequency and incident angle [13]. In this paper, we investigate the transmission coefficient from $40 \mathrm{GHz}$ to $50 \mathrm{GHz}$ with a fixed incident angle of 30 degrees. The other key parameters for the measurement system are given in Table 1, meanwhile, the dimensions and surface irregularities of MUT are recorded in Table 2, and we can appreciate that the marble is with rougher surface compared to the granite. In addition, all the measurements were continuously carried out under identical conditions.

For each material, two measurement campaigns were carried out: the first one is to obtain propagation coefficients for dielectric parameters' estimation by performing the reflection and transmission measurements; the second measurement is to collect received power of each $\mathrm{Rx}$ position to study the power angular profiles (PAP) for model parameterization and $\mathrm{RT}$ calibration. Moreover, the Tx and $\mathrm{Rx}$ are always orienting towards the center of MUT in each measurement, and the time gating technique is applied in the process of collecting measured data to decrease the impact from unwanted multipath components caused by other construction materials in the indoor environment, such as building floor, ceiling, or wall.

In reflection measurement as depicted in Figure 2(a), the Tx and Rx were positioned on the same side of MUT and at the distance of $0.5 \mathrm{~m}$ from the center of MUT to satisfy the farfield condition. The incident angle $\theta i$ was set to be 30 degrees, and the Rx was in the specular direction of Tx. The reference values of the reflection coefficient were measured by replacing MUT with a metal slab, which can be used to eliminate the undesired components including antenna radiation pattern and other effects from the propagation environment. 

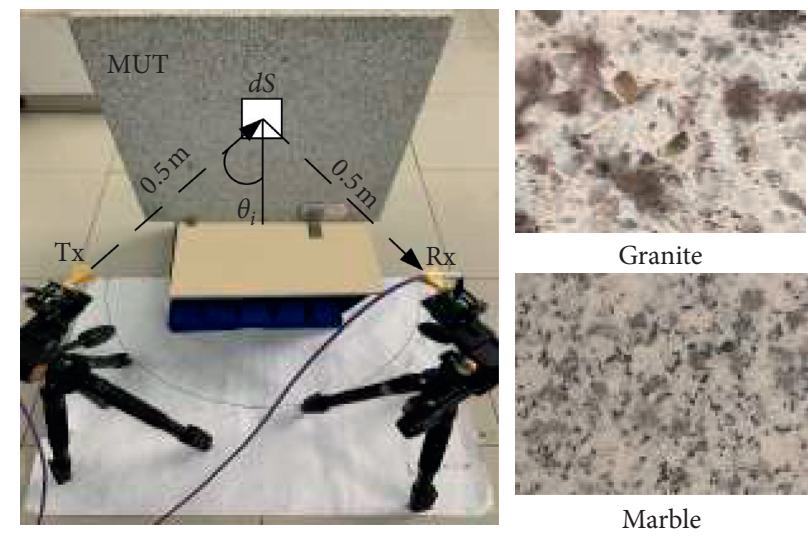

Figure 1: Measurement setup and snapshots of MUT.

TABle 1: Measurement system parameters.

\begin{tabular}{lc}
\hline Parameter & Value \\
\hline Center frequency & $45 \mathrm{GHz}$ \\
Bandwidth & $10 \mathrm{GHz}$ \\
Sweep frequency points & 1001 \\
Polarization type & Vertical \\
Tx power & $-2 \mathrm{dBm}$ \\
Measured radius & $0.5 \mathrm{~m}$ \\
Tx incident angle & $30^{\circ}$ \\
Rx step angle & $15^{\circ}$ \\
\hline
\end{tabular}

In transmission coefficient measurement (Figures 2(b) and 2(c)), the Tx and Rx were placed on the opposite sides of MUT and aligned with each other at the distance of $1 \mathrm{~m}$, and the MUT was placed in the middle of them. The reference measurement is also performed by removing MUT to obtain the transmission coefficient of air.

In the measurement for received power (Figure 2(d)), the Tx illuminated the center of MUT from a static incident direction where $\theta i=30^{\circ}$ while the $\mathrm{Rx}$ was moved along equispaced positions with the angular separation of $15^{\circ}$ on a semicircle arc with the radius of $50 \mathrm{~cm}$ from the center of MUT. Therefore, 11 receiver positions in total were measured for model parameterization and PAP analysis.

2.2. Dielectric Parameters Estimation. Considering the definition of propagation coefficients and power balance, it is
TABle 2: Dimensions and surface irregularities of MUT.

\begin{tabular}{lcccc}
\hline Material & $\begin{array}{c}\text { Length } \\
(\mathrm{cm})\end{array}$ & $\begin{array}{c}\text { Width } \\
(\mathrm{cm})\end{array}$ & $\begin{array}{c}\text { Thickness } \\
(\mathrm{cm})\end{array}$ & $\begin{array}{c}\text { Std. dev. } \\
(\mu \mathrm{m})\end{array}$ \\
\hline Granite & 76.8 & 42.0 & 1.52 & 0.361 \\
Marble & 60.0 & 60.0 & 2.36 & 0.928 \\
\hline
\end{tabular}

feasible to split the signal power after impinging the material surface into reflection, transmission, and scattering plus dielectric loss. Therefore, with the assumption of ideal power balance, the scattering coefficient $\mathrm{S}$ can be expressed as [7]

$$
S=\sqrt{1-\Gamma^{2}-T^{2}}=\sqrt{1-\left(\rho_{S} R\right)^{2}-T^{2}}
$$

where the theoretical values of reflection coefficient $\Gamma$ and transmission coefficient $T$ can be calculated by the complex permittivity $\eta$, which is composed of permittivity $\varepsilon$ as the real part and conductivity $\sigma$ constituting the imaginary. $\rho_{S}$ and $R$ are the reflection loss factor and the Fresnel reflection coefficient.

Thus, dielectric parameters of MUT can be estimated by tuning the values of $\varepsilon$ and $\sigma$ for the optimal match between the calculated and measured propagation coefficients, where the root-mean-square error (RMSE) of all frequency points is employed for quantitative evaluation, which can be written as

$$
\mathrm{RMSE}_{\text {coef }}=\sqrt{\frac{\sum_{i=1}^{M=1001}\left|\Gamma_{\text {mea. } \mathrm{i}}-\Gamma\right|^{2}+\sum_{i=1}^{M}\left|T_{\text {mea.i }}-T\right|^{2}+\sum_{i=1}^{M}\left|S_{\text {mea.i }}-S\right|^{2}}{M}},
$$

where $\Gamma_{\text {mea.i }}, T_{\text {mea.i }}$, and $S_{\text {mea.i }}$ are the measured reflection coefficient, transmission coefficient, and scattering coefficient, respectively, at $i$ th frequency point, and $M$ is the number of sweeping frequencies.

The theoretical values of $\Gamma$ and $T$ are derived from the well-known Fresnel formula, where the Gaussian rough surface model is adopted to calculate the scattering loss. Based on the measurement for propagation coefficients in
Section 2, the measured reflection coefficient $\Gamma_{\text {mea }}$ and transmission coefficient $T_{\text {mea }}$ can be expressed as

$$
\begin{gathered}
\Gamma_{\text {mea }}=\frac{S_{21, \mathrm{MUT}}}{S_{21, \text { metal }}} \\
T_{\text {mea }}=\frac{S_{21, \mathrm{MUT}}}{S_{21, \mathrm{air}}} .
\end{gathered}
$$




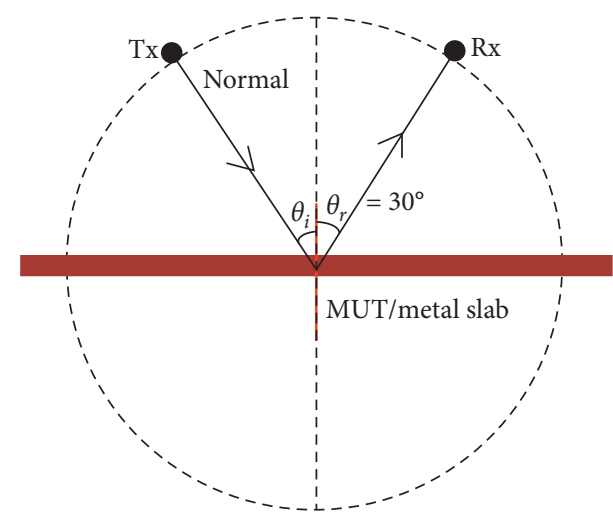

(a)

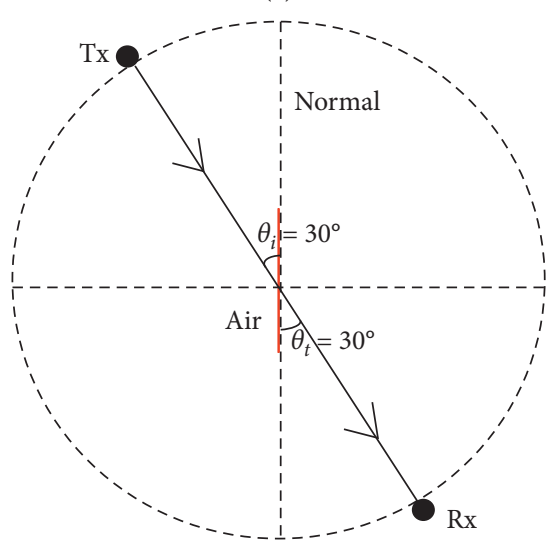

(c)

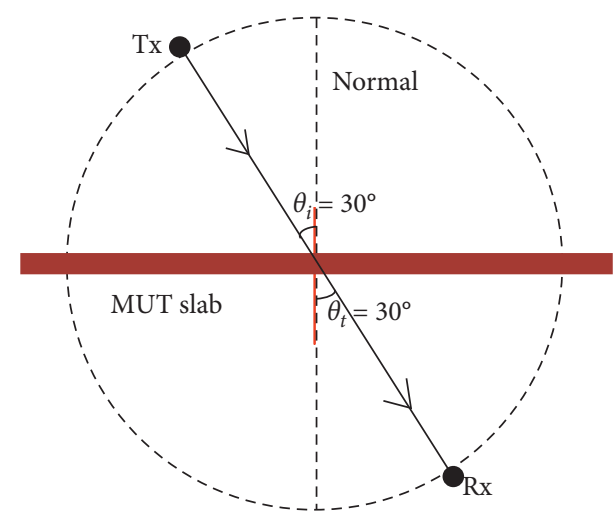

(b)

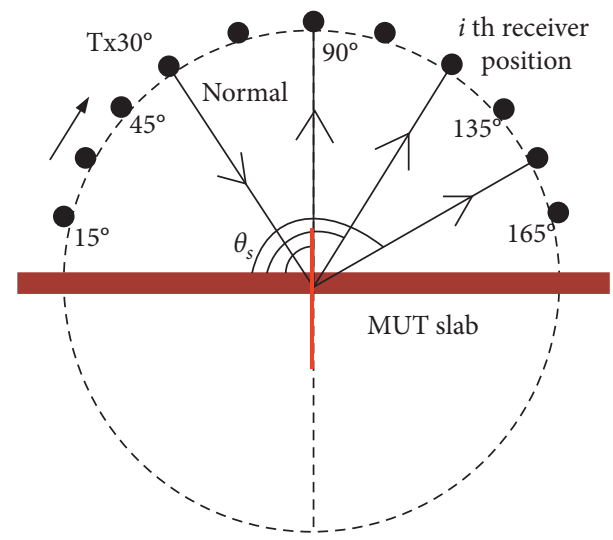

(d)

FIGURE 2: Measurement schemes for the reflection coefficient (a), transmission coefficient (b, c), and PAP (d).

Numerical comparison for the minimum RMSE between the two estimation methods and the optimal dielectric parameters of MUT are given in Table 3. Compared with the conventional tuning method (only estimates by reflection coefficient), the multicoefficient method in this paper can effectively improve the accuracy of material electromagnetic parameter estimation. And a more remarkable improvement can be observed for the granite; this is because the impact of diffuse scattering is greater due to its more protruding surface irregularity. Therefore, the accuracy of the parameter estimation of granite is improved by $43 \%$, while that of marble is only $5 \%$.

In Figure 3, the best-fit result in the frequency domain between the calculated and measured propagation coefficients is shown when the material is granite and $\theta i=30^{\circ}$ to give an example for determining the optimal dielectric parameters. The coefficients change periodically in $40 \mathrm{GHz}-50 \mathrm{GHz}$ frequency, and the resonant phenomenon takes place due to a series of waves undergoing multiple reflections and transmissions within the MUT which can be formally described using the internal successive reflection (ISR) model. Moreover, the estimated $\varepsilon$ and $\sigma$ are 5.37 and 0.19 for the granite and 5.57 and 0.37 for the marble, respectively.

\section{Directive Model Parameterization}

Once the measured scattering coefficient $S_{\text {mea }}$ and dielectric parameters of MUT have been obtained in Section 2, RT simulation for DS propagation can be performed to achieve the model parameterization. Meanwhile, measurements for the received power in the angle domain as shown in Figure 2(d) are conducted for tuning model parameters. Considering the measured received power distribution and surface roughness of MUT, the directive model is selected and the module of scattering electric field can be written as [7]

$$
\begin{aligned}
\left|\overline{E_{S}}\right|^{2} & =E_{S 0}^{2} \cdot\left(\frac{1+\cos \psi_{R}}{2}\right)^{\alpha_{R}} \\
& =\left(\frac{S K}{r_{i} r_{S}}\right)^{2} \frac{d S \cos \theta_{i}}{F_{\alpha_{R}}} \cdot\left(\frac{1+\cos \psi_{R}}{2}\right)^{\alpha_{R}}, \\
F_{\alpha_{R}} & =\frac{1}{2^{\alpha_{R}}} \sum_{j=0}^{\alpha_{R}}\left[\frac{2 \pi}{j+1}\left\{\cos \theta_{i} \cdot \sum_{\omega=0}^{(j-1) / 2}\left(\begin{array}{c}
2 \omega \\
\omega
\end{array}\right) \cdot \frac{\sin ^{2 \omega} \theta_{i}}{2^{2 \omega}}\right\}\left(1-(-1)^{j} / 2\right)\right] .
\end{aligned}
$$


TABLE 3: The minimum RMSE comparison between the conventional and proposed methods.

\begin{tabular}{|c|c|c|c|c|c|}
\hline \multirow[t]{2}{*}{ MUT } & \multirow[t]{2}{*}{ Conventional tuning method } & \multirow[t]{2}{*}{ Multicoefficient method } & \multirow[t]{2}{*}{ Enhanced accuracy (\%) } & \multicolumn{2}{|c|}{$\begin{array}{c}\text { Optimal } \\
\text { dielectric } \\
\text { parameters }\end{array}$} \\
\hline & & & & $\varepsilon_{r}$ & $\sigma$ \\
\hline Granite & 0.0849 & 0.0480 & 43 & 5.37 & 0.19 \\
\hline Marble & 0.0586 & 0.0559 & 5 & 5.57 & 0.37 \\
\hline
\end{tabular}

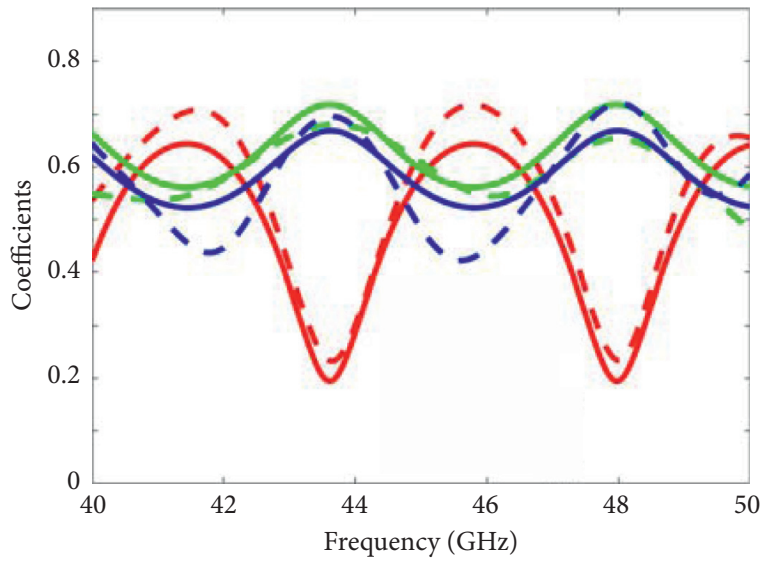

$\begin{array}{ll}- \text { Calculated } \Gamma & -- \text { Measured } T_{\text {mea }} \\ --- \text { Measured } \Gamma_{\text {mea }} & - \text { Calculated } S \\ \text { Calculated } T & --- \text { Measured } S_{\text {mea }}\end{array}$

Figure 3: Measured (dotted line) and calculated (solid line) propagation coefficients for the granite when $\theta i=30^{\circ}$.

Therefore, the DS patterns of MUT can be characterized by the parameter set:

$$
\Theta_{D} \triangleq\left\{S, \alpha_{R}, \theta_{i}, \Psi_{R}, r_{i}, r_{s}\right\},
$$

where $S$ is a scattering coefficient controlling the percentage of scattering power in the total power impinging on MUT element, $\psi \mathrm{R}$ is the angle between the direction of the reflected wave and the scattering direction, $\alpha \mathrm{R}$ is the scattering lobe width factor controlling the shape of the scattering pattern that greater $\alpha \mathrm{R}$ is corresponding to narrower scattering lobe, and the rest are angle and distance parameters, and $r_{i}$ and $r_{s}$ are the distances from the transmitting antenna and receiving antenna to the incident point on the surface of the material, respectively. Hence, the directive model parameterization is to determine the optimal values of $S$ and $\alpha \mathrm{R}$ for MUT. The tuning procedure for $\mathrm{S}$ and $\alpha \mathrm{R}$ is as follows based on the match between the simulated power $P_{\text {sim.i }}$ and measured power $P_{\text {mea.i }}$ at each received position, and the RMSE is adopted for evaluating the match which can be written as

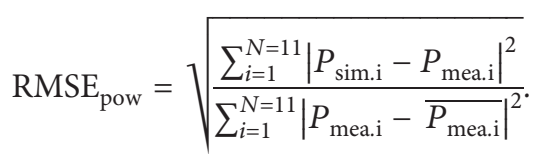

Step 1: set $S_{\text {mea }}$ as the initial value of $S$ and compare the RMSE with different values of $\alpha \mathrm{R}$ in the range $[1,10]$, which simplifies the procedure to determine the optimal $\alpha \mathrm{R}$ and no longer needs multiple comparisons for different $S$ in [11]

Step 2: adopt the optimal $\alpha \mathrm{R}$ and vary $S$ around the $S_{\text {mea }}$ to obtain the appropriate range of $S$ according to RMSE

Based on the proposed method, model parameterization has been performed for MUT. The optimal results of model parameterization are determined through curve analysis and numerical comparison for RMSE. Tuning results, respectively, at $40 \mathrm{GHz}, 45 \mathrm{GHz}$, and $50 \mathrm{GHz}$ for MUT are presented in Figures 4 and 5. It can be observed that the variation trends of RMSE are clearly distinguished for each MUT at the presented frequency points, but they are acceptable because of their different dielectric characteristics and surface roughness.

In Figure 4, larger values of $\alpha \mathrm{R}$ are found to be more suitable for describing the DS propagation phenomenon. It can be explained that scattering rays caused by the MUT surface are mainly concentrated around the specular direction, which validates the selection for the directive model rather than the other two models proposed in [7]. In addition, the tuning results for $S$ are presented in Figure 5 based on the optimal $\alpha \mathrm{R}$, where the RMSE values show approximate parabola variation and the lowest point is corresponding to the optimal $\mathrm{S}$ for specific frequency and MUT.

Numerical results of model parameterization including the optimal $\alpha \mathrm{R}, \mathrm{S}$, and fitting errors are recorded in Table 4 . The optimal values of $\alpha \mathrm{R}$ equal to 10 for both MUT at presented frequency points. Fluctuation within 0.1 around the optimal value can be accepted as the proper range of $S$ since the very slight RMSE differences. The values of $S$ for the marble are distinctly larger than those for the granite, which is corresponding to the more severe surface irregularity of the marble. Besides, it can be observed that the optimal $\mathrm{S}$ is slightly smaller than measured values because the dielectric loss power has been counted in measured scattering power, which causes the value of $S_{\text {mea }}$ to be larger than the actual. Moreover, this analysis can be demonstrated by the fact that the difference is more remarkable for the marble, which generates more dielectric loss due to larger conductivity and inherent thickness.

\section{Performance Evaluation}

For the sake of performance evaluation for the proposed parameterization methodology in RT calibration, the overall workflow is shown in Figure 6. Note that the environment modeling and configuring deployment corresponding to the measurement is the precondition for RT simulation. In order 


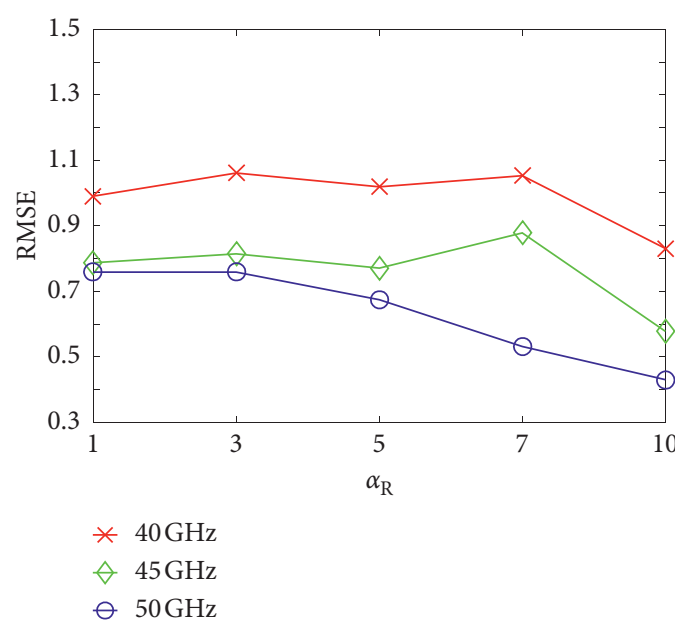

(a)

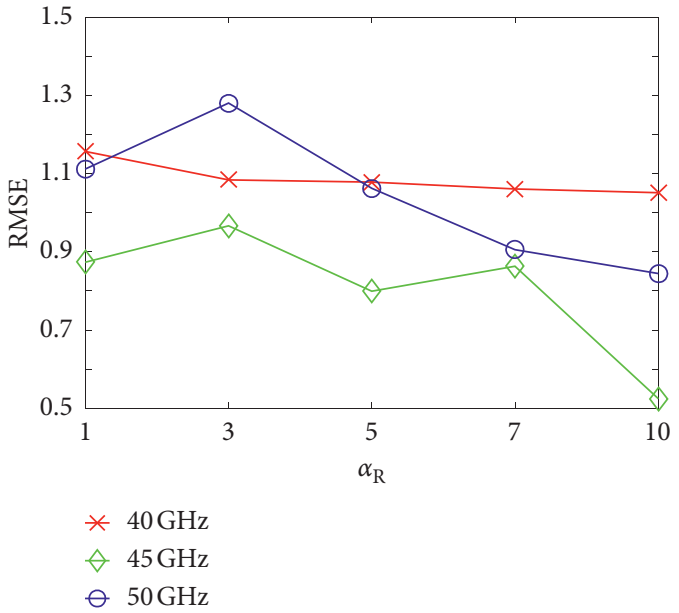

(b)

Figure 4: RMSE comparisons for different values of $\alpha \mathrm{R}$ for the granite (a) and marble (b).

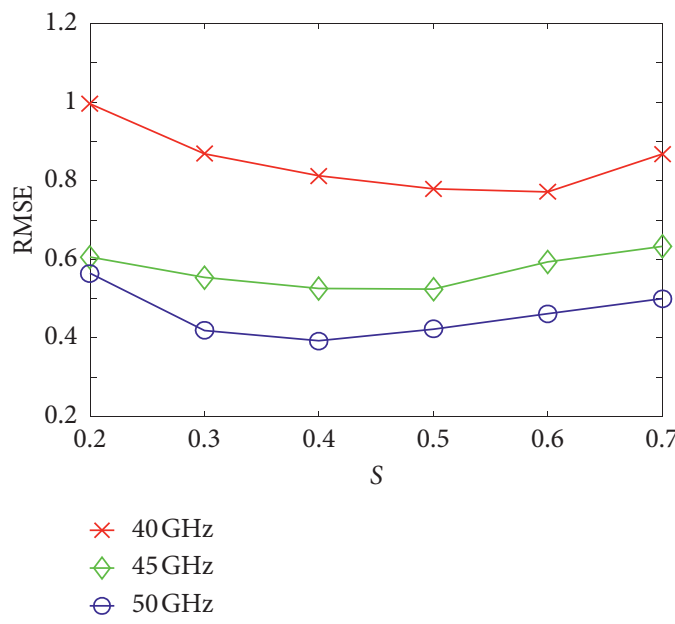

(a)

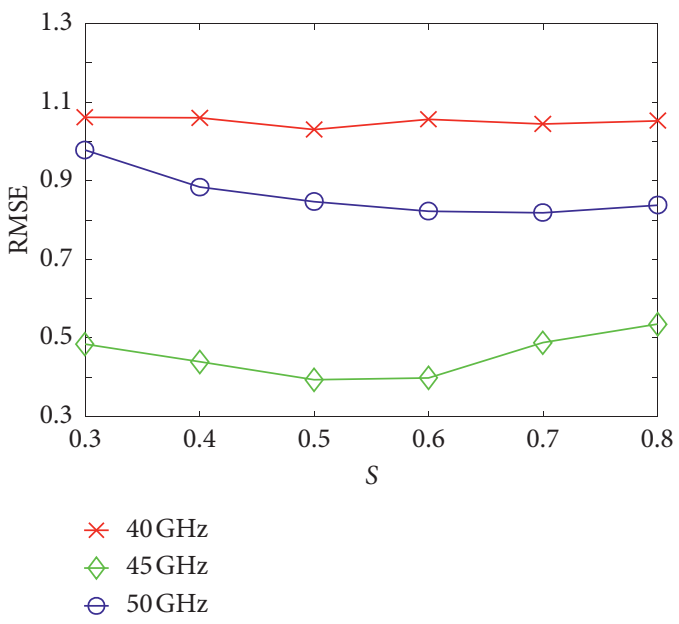

(b)

FIgURE 5: RMSE comparisons for different values of S for the granite (a) and marble (b).

TABLE 4: Summary of parameterization results.

\begin{tabular}{lccccc}
\hline \multirow{2}{*}{ MUT } & Frequency $(\mathrm{GHz})$ & \multicolumn{4}{c}{ Optimal } \\
& & $\alpha_{R}$ & $S$ & & \\
\hline \multirow{3}{*}{ Granite } & 40 & 10 & $0.5-0.6$ & 0.62 & 0.7712 \\
& 45 & 10 & $0.4-0.5$ & 0.56 & 0.5244 \\
& 50 & 10 & $0.3-0.5$ & 0.52 & 0.3926 \\
\hline \multirow{3}{*}{ Marble } & 40 & 10 & $0.5-0.6$ & 0.76 & 1.0299 \\
& 45 & 10 & $0.5-0.6$ & 0.78 & 0.3933 \\
& 50 & 10 & $0.6-0.7$ & 0.82 & 0.8183 \\
\hline
\end{tabular}

to limit the running time and computational burden of RT simulation, the maximum numbers of reflection, transmission, and diffraction for one ray path are set to be 3,1 , and 1 , respectively. Then, the dielectric parameters estimated in Section 2 are used for the parameter initialization of MUT, which is different from the method in [7]. Moreover, the directive model is parameterized to enable RT calibration, and the RMSE between the measured and simulated power from the tuning procedure is used as the evaluation criterion to determine the optimal model parameters.

Each ray receives power and the channel characteristic parameters are obtained from RT simulation in the 40-50 GHz band. The parameters for the environment description are set to the value used in Table 1. All paths are modeled by reflection, diffraction, and penetration based on the geometrical optics (GO) and uniform theory of diffraction (UTD) using the software Wireless InSite by REMCOM. The main RT simulation process is as follows:

(i) Step 1: Propagation environment modeling. The indoor millimeter-wave propagation environment model in RT simulation is presented in Figure 7(a), which is modeled according to the actual measurement scenario. Dimension and the location of 


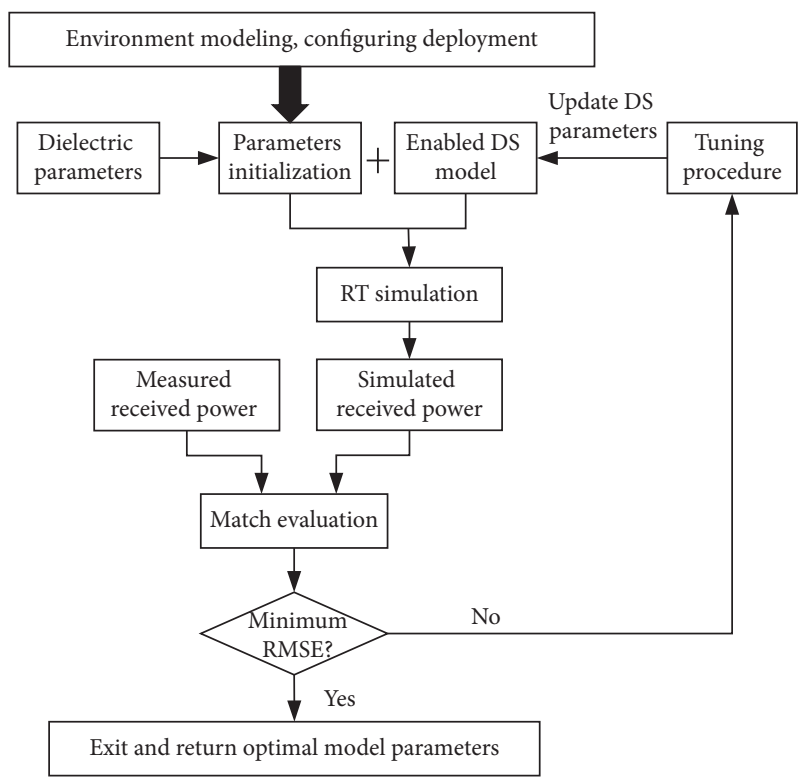

FIGURE 6: Workflow of RT calibration based on measurement and DS model parameterization.

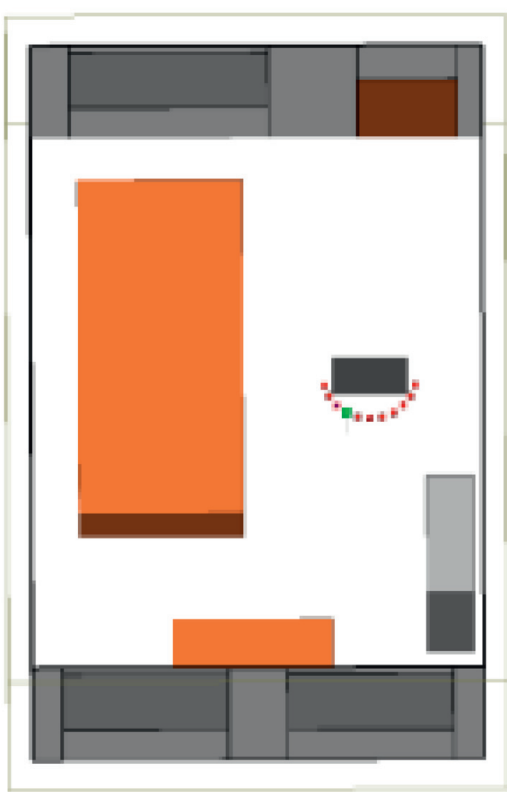

(a)

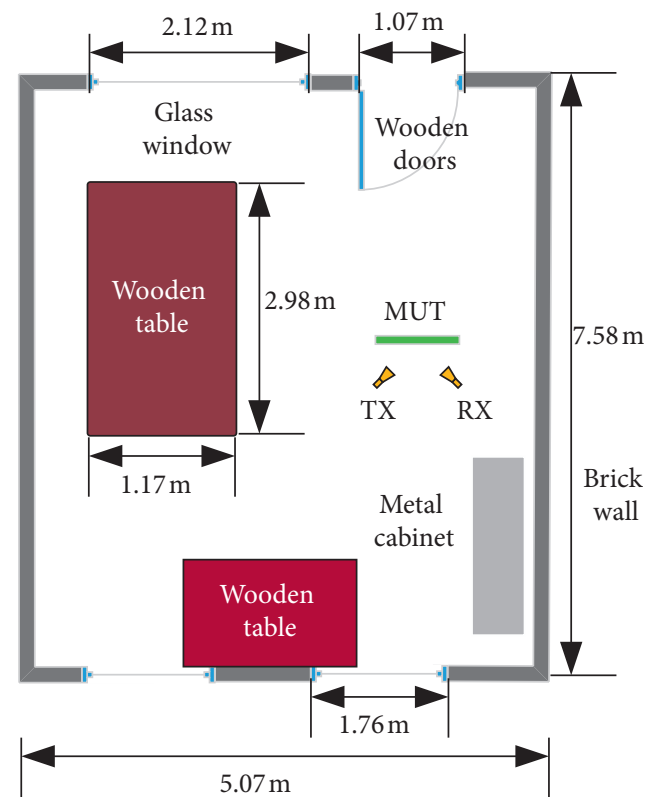

(b)

FIgURE 7: RT simulation model (a) and the actual size of the propagation environment (b).

objects, including wall, floor, ceiling, door, window, wooden table, metal cabinet, and measured material, are shown in Figure 7(b). With the exception of MUT, the standard electromagnetic characteristic parameters of typical indoor materials in the millimeter-wave band are set, as recorded in Table 5.

Step 2: Deployment of receiving and transmitting antenna. The Tx and $\mathrm{Rx}$ were placed $67 \mathrm{~cm}$ above the ground and the parameters such as aperture size and
TABLE 5: Electromagnetic parameters of various materials.

\begin{tabular}{lccc}
\hline Material & $\begin{array}{c}\text { Relative dielectric } \\
\text { constant } \varepsilon_{r}\end{array}$ & Conductivity $\sigma$ & $\begin{array}{c}\text { Thickness } d \\
(\mathrm{~cm})\end{array}$ \\
\hline Wood & 1.99 & 0.378 & 3.00 \\
Glass & 6.27 & 0.567 & 0.30 \\
Metal & 1.00 & $10^{7}$ & - \\
Floor & 3.66 & 1.113 & 3.00 \\
Ceiling & 1.50 & 0.059 & 0.95 \\
Brick & 4.44 & 0.001 & 12.50 \\
\hline
\end{tabular}


TABLE 6: Calculation methods of different propagation mechanisms.

\begin{tabular}{lc}
\hline Propagation mechanism & Calculation method \\
\hline LOS & Friis formula \\
Reflection & Fresnel formula \\
Transmission & Fresnel formula \\
Diffraction & Uniform theory of diffraction \\
Scattering & Directive model \\
\hline
\end{tabular}

transmitting power of the horn antenna are set according to the configuration of the measurement platform. The point type and arc type deployment modes are, respectively, adopted for the transmitting antenna (green) and receiving antenna (red) in Figure 7(a).

Step 3: Set propagation characteristics of the MUT. The structure of MUT is set as a single-layer medium, and the best estimation value obtained in Section 3 is used for the electromagnetic parameters. The directive model is selected as the diffuse scattering model, and the directive model parameters are set according to the corresponding tuning method in Section 3.

Step 4: Set up the RT simulation domain and ray propagation model. The simulation domain is created by the automatic fitting of the whole propagation environment model boundary, and the X3D model is selected as the ray propagation model to support diffuse scattering propagation calculation. Table 6 gives the calculation of different propagation mechanisms in RT simulation.

After the RT simulation, the visible propagation path and receive power for marble are shown in Figure 8, in which 104 propagation paths are included, different colors of path correspond to different values of power, the power dynamic range of receiver at 120 degrees is $-30.6 \mathrm{dBm}$ to $-172.9 \mathrm{dBm}$, and the delay spread and time of arrival channel characteristic also can be obtained in output. In order to verify the accuracy improvement of RT simulation with the proposed DS model parameterization methodology and the feasibility for both MUT, the optimal match results of PAP are depicted in Figures 9 and 10, where the specular power without scattering model (black dashed line) and received power using the directive model without the optimal model parameters $\alpha_{R}$ and $S$ (green solid line) are presented as comparisons.

It can be observed that simulated power distribution with the optimal model parameters presents better agreement with the measured values than other simulated results. Furthermore, it is worth noting that the measured received power is approximately $20 \mathrm{dBm}$ stronger than simulated results without the DS model in nonspecular directions, which demonstrates that the significant proportion of DS in mmWave propagation components can no longer be neglected. Therefore, the optimal DS model parameters of materials are essential for high-performance RT simulation in mmWave bands to achieve the accurate channel analysis.

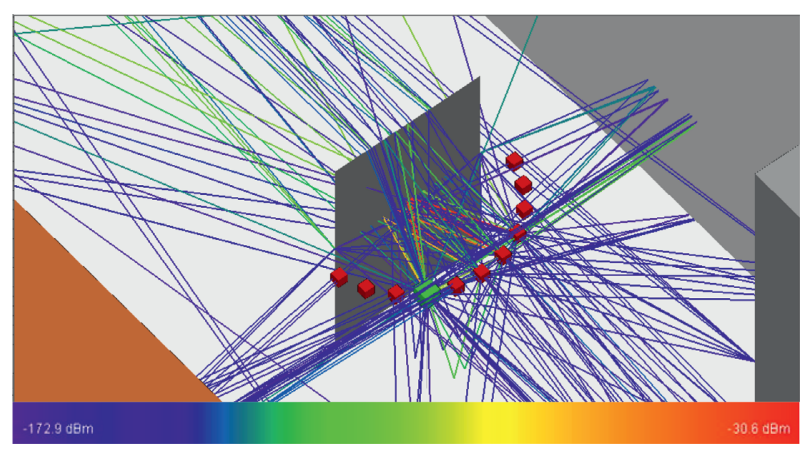

FIGURE 8: Visible propagation path and receive power for marble.

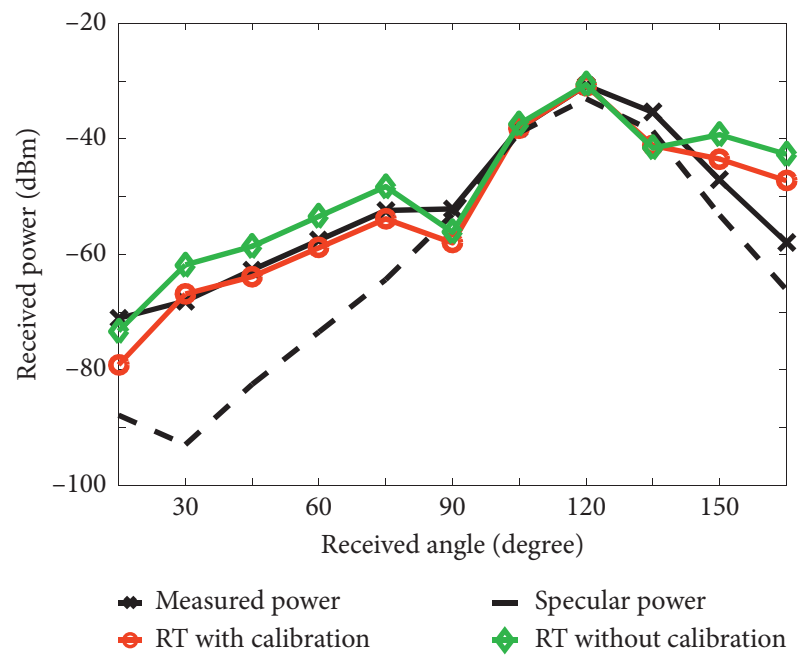

Figure 9: Measured and simulated PAP for the granite at $50 \mathrm{GHz}$.

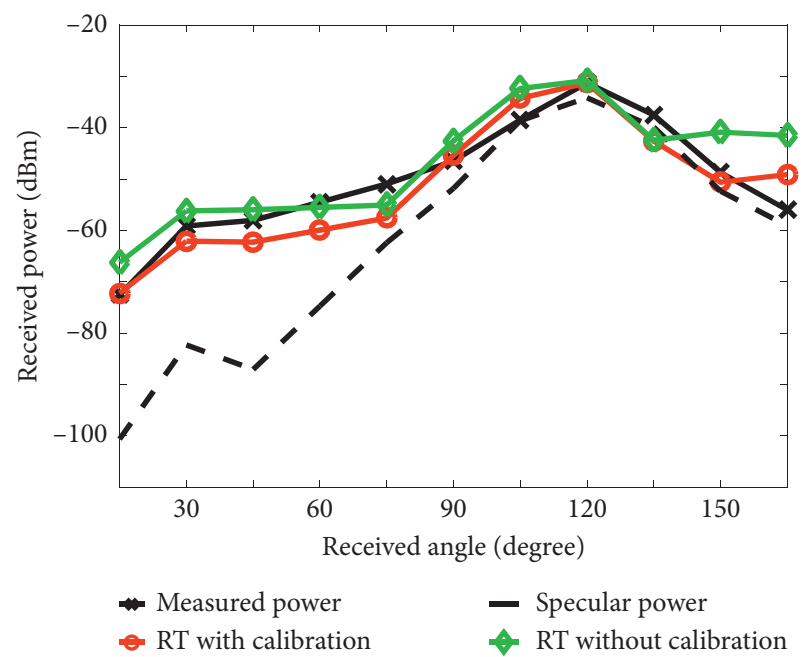

Figure 10: Measured and simulated PAP for the marble at $45 \mathrm{GHz}$.

\section{Conclusions}

In this paper, the optimal scattering model parameters of MUT are determined and embedded into RT simulation for the DS propagation at mmWave frequencies. The complex permittivities of granite and marble are estimated through 
propagation coefficients match between the measured and calculated values, where the scattering coefficient is derived from the ideal power balance. A remarkable conclusion can be drawn from comparative results between the measured and simulated PAP for MUT where calibrated RT simulation with the optimal DS model parameters enables higher accuracy for predicting mmWave propagation. Moreover, the proposed DS model parameterization methodology is validated to be suitable for materials with different surface irregularities and inherent properties at mmWave frequencies. Our future work will be focused on building the database of DS model parameters for different types of construction materials in various mmWave bands by conducting extensive measurement and simulation campaigns. Furthermore, the effects of polarization properties on scattering should be investigated.

\section{Data Availability}

The data used to support the findings of this study are included within the article.

\section{Conflicts of Interest}

The authors declare that they have no conflicts of interest.

\section{Acknowledgments}

This work was supported in part by the National Natural Science Foundation of China under Grant 61801062 and Grant 61801063, the State Key Laboratory of Millimeter Waves under Grant K202032 and Grant K202005, the China Postdoctoral Science Foundation under Grant 2019M653826XB, and the Chongqing Research Program of Basic Research and Frontier Technology under Grant cstc2019jcyj-msxmX0288.

\section{References}

[1] A. V. Alejos, M. G. Sánchez, and I. Cuiñas, "Measurement and analysis of propagation mechanisms at $40 \mathrm{GHz}$ : viability of site shielding forced by obstacles," IEEE Transactions on Vehicular Technology, vol. 57, no. 6, pp. 3369-3380, Nov. 2008.

[2] F. Fuschini, E. M. Vitucci, M. Barbiroli, G. Falciasecca, and V. Degli-Esposti, "Ray tracing propagation modeling for future small-cell and indoor applications: a review of current techniques," Radio Science, vol. 50, no. 6, pp. 469-485, 2015.

[3] F. Mani, F. Quitin, and C. Oestges, "Directional spreads of dense multipath components in indoor environments: experimental validation of a ray-tracing approach," IEEE Transactions on Antennas and Propagation, vol. 60, no. 7, pp. 3389-3396, 2012.

[4] M. Shafi, J. Zhang, H. Tataria et al., "Microwave vs. millimeterwave propagation channels: key differences and impact on 5G cellular systems," IEEE Communications Magazine, vol. 56, no. 12, pp. 14-20, 2018.

[5] E. M. Vitucci, J. Chen, V. Degli-Esposti, J. S. Lu, H. L. Bertoni, and $\mathrm{X}$. Yin, "Analyzing radio scattering caused by various building elements using millimeter-wave scale model measurements and ray tracing," IEEE Transactions on Antennas and Propagation, vol. 67, no. 1, pp. 665-669, 2019.
[6] D. Solomitckii, Q. C. Li, T. Balercia et al., "Characterizing the impact of diffuse scattering in urban millimeter-wave deployments," IEEE Wireless Communications Letters, vol. 5, no. 4, pp. 432-435, Aug. 2016.

[7] V. Degli-Esposti, F. Fuschini, E. M. Vitucci, and G. Falciasecca, "Measurement and modelling of scattering from buildings," IEEE Transactions on Antennas and Propagation, vol. 55, no. 1, pp. 143-153, Jan. 2007.

[8] D. He, B. Ai, M. Schmieder et al., "Influence analysis of typical objects in rural railway environments at $28 \mathrm{GHz}$," IEEE Transactions on Vehicular Technology, vol. 68, no. 3, pp. 2066-2076, 2019.

[9] L. Minghini, R. D’Errico, V. Degli Esposti, and E. M. Vitucci, "Electromagnetic simulation and measurement of diffuse scattering from building walls," in Proceedings of the 8th European Conference on Antennas and Propagation (EuCAP), pp. 1298-1302, The Hague, Netherlands, April 2014.

[10] S. Ju, S. H. A. Shah, M. A. Javed et al., "Scattering mechanisms and modeling for Terahertz wireless communications," in Proceedings of the ICC 2019-2019 IEEE International Conference on Communications (ICC), pp. 1-7, Shanghai, China, May 2019.

[11] J. Pascual-García, J. M. Molina-García-Pardo, M. T. MartínezInglés, J. V. Rodríguez, and N. Saurín-Serrano, "On the importance of diffuse scattering model parameterization in indoor wireless channels at mm-wave frequencies," IEEE Access, vol. 4, pp. 688-701, 2016.

[12] H. Tian, X. Liao, Y. Wang et al., "Effect level based parameterization method for diffuse scattering models at millimeterwave frequencies," IEEE Access, vol. 7, pp. 93286-93293, 2019.

[13] I. T. Union, "Effects of building materials and structures on radiowave propagation above $100 \mathrm{MHz}$," in Standard ITU-R PElectronic Publication, Geneva, Switzerland, 2015. 